\title{
A resource-poor developmental diet reduces adult aggression in male Drosophila melanogaster
}

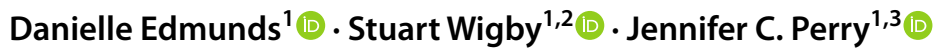

Received: 13 January 2021 / Revised: 26 June 2021 / Accepted: 30 June 2021 / Published online: 22 July 2021

(c) The Author(s) 2021

\begin{abstract}
Aggressive behaviours occur throughout the animal kingdom and agonistic contests often govern access to resources. Nutrition experienced during development has the potential to influence aggressive behaviours in adults through effects on growth, energy budgets and an individual's internal state. In particular, resource-poor developmental nutrition might decrease adult aggression by limiting growth and energy budgets, or alternatively might increase adult aggression by enhancing motivation to compete for resources. However, the direction of this relationship — and effects of developmental nutrition experienced by rivals-remains unknown in most species, limiting understanding of how early-life environments contribute to variation in aggression. We investigated these alternative hypotheses by assessing male-male aggression in adult fruit flies, Drosophila melanogaster, that developed on a low-, medium- or high-resource diet, manipulated via yeast content. We found that a low-resource developmental diet reduced the probability of aggressive lunges in adults, as well as threat displays against rivals that developed on a low-resource diet. These effects appeared to be independent of diet-related differences in body mass. Males performed relatively more aggression on a central food patch when facing rivals of a low-resource diet, suggesting that developmental diet affects aggressive interactions through social effects in addition to individual effects. Our finding that resource-poor developmental diets reduce male-male aggression in D. melanogaster is consistent with the idea that resource budgets mediate aggression and in a mass-independent manner. Our study improves understanding of the links between nutrition and aggression.
\end{abstract}

\section{Significance statement}

Early-life nutrition can influence social behaviours in adults. Aggression is a widespread social behaviour with important consequences for fitness. Using the fruit fly, Drosophila melanogaster, we show that a poor developmental diet reduces aspects of adult aggressive behaviour in males. Furthermore, males perform more aggression near food patches when facing rivals of poor nutrition. This suggests that early-life nutrition affects aggressive interactions through social effects in addition to individual effects.

Keywords Aggression $\cdot$ Development $\cdot$ Diet $\cdot$ Drosophila melanogaster $\cdot$ Nutrition

\section{Introduction}

Communicated by M. Giurfa.

Jennifer C. Perry

jennifer.perry@uea.ac.uk

1 Department of Zoology, University of Oxford, Oxford, UK

2 Department of Evolution, Ecology, and Behaviour, Institute of Infection, University of Liverpool, Veterinary \& Ecological Sciences, Liverpool, UK

3 School of Biological Sciences, University of East Anglia, Norwich NR4 7TJ, UK
Aggression is widespread amongst animals (e.g. mammals, Sinn et al. 2008; birds, Johnsen and Zuk 1995; fish, Neat et al. 1998; Seebacher et al. 2013; invertebrates, Brown et al. 2007; Elias et al. 2010), including humans, where aggressive behaviours have detrimental effects on societies (Blanchard and Blanchard 2003; Sluyter et al. 2003; Georgiev et al. 2013). Success in aggressive contests can provide superior access to critical reproductive resources such as food, territories and mates (Clutton-Brock and Albon 1979; Hoffman 1987; Huntingford et al. 2012; Georgiev et al. 2013; Belenioti and 
Chaniotakis 2020), but contests come with costs, including physical damage, time and energy expenditure and increased predation risk (Haley 1994; Neat et al. 1998; Briffa and Sneddon 2007). Individuals often display temporary, reversible changes in aggressive behaviours throughout life (Huntingford et al. 2012; Georgiev et al. 2013), and much of this variation is likely to result from differences in an individual's environment (Dochtermann et al. 2015; Han and Dingemanse 2017; Bath et al. 2021). Understanding the ecological factors that determine aggressive behaviour can help to elucidate its evolution and consequences.

Nutrient availability and quality are key components of an individual's environment and can shape behavioural strategies expressed throughout life (Lihoreau et al. 2015). In many species, early life is key for nutrient acquisition, and the balance of nutrients in this critical period can have profound effects on body mass, resource allocation to adult traits, and internal state (Royle et al. 2005; Amitin and Pitnick 2007; Zikovitz and Agrawal 2013; Lihoreau et al. 2015; Pillay et al. 2016; Han and Dingemanse 2017). These effects can determine the relative ability (i.e. resource-holding potential) and motivation (i.e. resource valuation, the value of a contested resource; Elias et al. 2010; Stockermans and Hardy 2013; Gruber et al. 2016) to invest in aggressive contests and the fitness pay-offs from doing so. Thus, individuals should benefit from moderating aggression adaptively in response to nutritionally derived cues experienced in early life (Scharf 2016). Consistent with this prediction, there is evidence that early-life diet influences levels of aggression and antisocial behaviours in humans and non-human vertebrates (Wallner and Machatschke 2009).

However, the net effect of developmental nutrition on resource-holding potential and resource valuation-and hence the direction of the relationship between developmental nutrition and aggression-remains unclear. Resourcerich developmental nutrition often increases adult body mass, and larger individuals are more likely to initiate and win aggressive contests in many species (Hoffman 1987; Shackleton et al. 2005; Briffa and Sneddon 2007; Brown et al. 2007; Bath et al. 2018). Likewise, high nutrient availability during development can increase relative resource allocation to traits (such as weapons) that enhance aggressive ability (Monaghan 2008; Colasurdo et al. 2009). Alternatively, resource-rich developmental nutrition might decrease aggressive motivation and the fitness benefits an individual gains from attaining a resource, through effects on the internal state (Arnott and Elwood 2008; Elias et al. 2010; Bath et al. 2018). For example, a lack of nutritional resources can increase motivation to attain food (Arnott and Elwood 2008). Likewise, if resource-poor nutritional conditions decrease lifespan (Good and Tatar 2001; Tigreros 2013), then they might increase motivation to attain access to breeding sites and mates (i.e. terminal investment; Clutton-Brock 1984; Krams et al. 2015; Moatt et al. 2016).

The existing studies that have investigated the relationship between developmental nutrition and aggression report contrasting responses across species. In the African striped mouse, Rhabdomys dilectus chakae, early-life protein deficiency leads to increased aggressive behaviour (Pillay et al. 2016), and low food availability increases aggressive lunging in the monarch caterpillar Danaus plexippus (Collie et al. 2020). However, in the southern field cricket Gryllus bimaculatus, a high-protein developmental nutrition increases aggression (Han and Dingemanse 2017), and aggression is higher in Argentine ant colonies (Linepithema humile) that develop on carbohydrate-rich diets (Grover et al. 2007). Furthermore, variation in developmental nutrition amongst individuals can influence aggressive interactions by generating asymmetries in fighting ability amongst rivals (Parker 1974; Briffa and Sneddon 2007; Asahina 2017), but few studies have evaluated the effects of developmental nutrition on both focal and rival individuals. Hence, the strength and direction of developmental nutrition effects on adult aggression remain largely unknown.

We used the fruit fly, Drosophila melanogaster, to investigate how the diet experienced during development influences adult male aggressive strategies. Drosophila melanogaster serves as an important model organism for both aggression and nutrition. Male D. melanogaster engage in frequent contests over mates and food patches, and success in contests influences mating success (Kravitz and Fernandez 2015). Access to food patches can provide increased nutrition, but, because males display only limited adult feeding (Carvalho et al. 2006), access to food patches largely provides access to mates (Dow and von Schilcher 1975; Hoffman 1987; Hoffmann 1987; Chen et al. 2002). Aggressive behaviours range from wing threat displays and fencing spars with forelegs to lunging, the principal aggressive behaviour, in which a male rears up and thrusts his upper body at his opponent (Hoffmann 1987; Chen et al. 2002; supplementary table 1). As a holometabolous insect with a juvenile food-acquiring stage distinct from the adult stage, the nutrition received during early life is critical to development in D. melanogaster (Boggs 1981). For example, early-life nutrition strongly impacts viability, body mass and post-copulatory reproductive traits (e.g. Gebhardt and Stearns 1993; Bross et al. 2005; McGraw et al. 2007). However, despite the wealth of knowledge on both nutrition and agonistic contests in this species, the direct link between developmental nutrition and adult aggressive behaviours has not been fully explored. Because D. melanogaster is a leading model organism for the neurobiology and physiology of aggression (Asahina 2017), understanding the relationship between developmental nutrition and aggression in this species would help to establish a system for in-depth investigation of the mechanisms linking nutrition and aggression. 
We subjected male flies to low-, medium- or highresource diets during larval development by manipulating yeast levels and then measured their adult aggressive behaviours. Yeast is an important source of protein, which is a key component of developmental nutrition for herbivorous insects that can be limiting under natural conditions (Lihoreau et al. 2015; Han and Dingemanse 2017). To test the effects of both an individual's diet and the diet of its rival, we set up contests between pairs of males from all combinations of diet treatment in a fully factorial experimental design. We predict that if developmental diet influences aggression through effects on resource-holding potential, then highresource developmental diets will increase adult aggression, whereas if developmental diet influences aggression through increased resource valuation, then low-resource developmental diets will increase aggression.

\section{Methods}

\section{Experimental flies}

All flies were derived from an outbred wild-type Dahomey stock population that has been maintained since 1970 in cages with overlapping generations (Carazo et al. 2015). Our use of an outbred population gives us the ability to explore variation in multiple aggressive traits. Stock populations were maintained on a standard molasses-based media (supplementary table 2). Fly husbandry was carried out at $25^{\circ} \mathrm{C}$ on a 12:12 h light:dark cycle.

To generate experimental males, we collected eggs from the stock population using grape-agar plates smeared with yeast, transferred them at a standard density (50 eggs/vial) to vials containing food media (low, medium or high protein) and incubated them until eclosion. For ease of diet manipulation, we modified a simple sugar-yeast-agar medium (yeast:sugar ratio of 2:1) to create a 'low' (L; $10 \%$ yeast), 'medium' (M; 20\% yeast) and 'high' resource media $(\mathrm{H} ; 120 \%$ yeast; supplementary table 3$)$. Yeast is the main source of protein in D. melanogaster diets and also contains micronutrients (e.g. vitamins, nucleic acids and cholesterol; Sang 1978). The optimal protein to carbohydrate ratio for male D. melanogaster larvae is between $1.5: 1$ and $2: 1$ (Rodrigues et al. 2015; Jang and Lee 2018), suggesting that our medium- and low-yeast media were substantially suboptimal in protein and that our high-yeast media contained slightly above optimal protein. We refer to these treatments as resource-poor or -rich to reflect that yeast manipulation alters protein, caloric and micronutrient content. Preliminary tests showed that these differences in yeast level were sufficient to generate differences in developmental duration and adult body mass. Developmental duration was extended as protein quantity was reduced, so egg collections for each treatment were staggered to synchronise adult eclosion.

We collected adult males using ice anaesthesia within $6 \mathrm{~h}$ of eclosion to ensure virginity, and transferred them to vials containing a standard molasses-based media, housing them individually to prevent the formation of social hierarchies that might influence aggressive behaviour (Penn et al. 2010; Trannoy et al. 2016). To differentiate males in behavioural trials, we painted each male with a small dot of red or white acrylic paint on the dorsal thorax between 1 and 4 days posteclosion. Similar paint treatments had no detectable effect on behaviour in previous studies (Nilsen et al. 2004; Morimoto et al. 2016).

\section{Behavioural trials}

Before trials, we deprived flies of food for $2 \mathrm{~h}$ in vials containing moist cotton wool to prevent desiccation. We then transferred pairs of males via gentle aspiration into observation chambers (20-mm diameter, 5-mm depth) containing a food patch of molasses-based media combined with yeast paste (5-mm diameter), a standard protocol for assessing aggression in D. melanogaster (Dierick 2007). In each pair, we arbitrarily designated one male as the focal male and the other as the rival male. We paired males in all combinations of treatments in a fully factorial design with 20-21 pairs per combination (supplementary table 4). To avoid confounding effects of paint colour, we painted half of the focal males red and the other half white. After a 5-min acclimatisation period, we recorded behaviour using a video camera (Toshiba Camileo X400) for $15 \mathrm{~min}$. We conducted behavioural trials between 2 and $7 \mathrm{~h}$ Zeitgeber time over 4 days, with each individual fly trialled only once.

A single observer blind to treatment scored the videos using JWatcher (v. 1.0, Blumstein and Daniel 2007) and recorded the duration and number of occurrences of five aggressive behaviours (fencing, chasing, tussling, lunging and wing threat; supplementary table 1) for each focal and rival male. We also recorded the total locomotion duration and whether behaviours were performed on or off the food patch to allow testing for differences in locomotion and food patch access.

After trials, we froze and weighed males to assess the influence of nutrition on body mass. We weighed flies immediately after freezing to assess their mass during trials (wet mass), and weighed them again after they were dried for $48 \mathrm{~h}$ at $60{ }^{\circ} \mathrm{C}$ (dry mass).

\section{Statistical analyses}

We performed analyses in $\mathrm{R}$ version 3.6.2 (2019-12-12). We expressed behavioural data as total durations (in seconds) or number of bouts of each behaviour. To test the influence of focal male diet, rival male diet and their interaction on 
focal male aggression, we first analysed lunging behaviour, the principal form of aggression displayed by male $D$. melanogaster (e.g. Hoyer et al. 2008; Asahina et al. 2014). Because lunging data were zero-inflated (101 of 187 focal males lunged), we analysed the influence of developmental diet on the likelihood of lunging in a binomial general linear model, and then on lunge number (within the subset of flies that did lunge) using a negative binomial general linear model.

Wing threat behaviour is a non-contact threat component of male aggressive behaviour. We analysed the influence of developmental diet on the number of focal male wing threats using a negative binomial distribution.

We then analysed the influence of developmental diet on total aggression duration, summed over lunging, fencing, tussling and wing threat, using a general linear model. Because our findings for total aggression differed from those for lunging, we secondarily explored total aggression further by conducting general linear models for the duration of fencing, and likelihood and duration of chasing independently. Chasing data were also zero-inflated (58 of 187 focal males chased), so we analysed the influence of diet on the likelihood of chasing in a binomial general linear model, and then on chase durations in the subset of flies that displayed chasing using a Gaussian general linear model. Because there were only two incidents of tussling, we did not analyse tussling separately. Because diet might affect locomotion, which might impact aggressive encounters, we assessed the influence of focal and rival diet on locomotion in a linear model.

To assess the interdependence of behaviour of the two individuals in a pair, we used a chi-square test of independence to assess whether the likelihood of a focal male lunging was related to the likelihood of his rival lunging. We then assessed the influence of rival lunge number on focal lunge number in the subset of males that lunged, and the influence of rival total aggression duration on focal total aggression duration amongst all males in linear models. To test whether this relationship was influenced by developmental diet, we performed chi-square tests of independence for each focal developmental diet treatment separately, and we ran an additional model including focal and rival diet and their interaction with rival aggression on focal total aggression.

In male D. melanogaster, food patches provide access to mates and males aggressively defend these sites (Markow 1988; Hoffman and Cacoyianni 1990; Lim et al. 2014). To test for differences in food patch occupancy, we analysed the influence of focal and rival developmental diet and their interactions on the amount of time focal individuals spent on the food patch using linear models. To evaluate how diet affects aggressive behaviour around food patches, we assessed the influence of focal and rival developmental diet on the proportion of aggressive behaviour that a focal individual performed on the food patch using general linear models fitted with the quasibinomial distribution.

We used diagnostic plots to assess model fit, and where data were over-dispersed, we used square root or log transformations to avoid violating the assumptions of parametric statistics. We included day as a fixed factor (Harrison et al. 2018) and Zeitgeber time as a covariate in all models to account for temporal variation in behaviour. We initially assessed models using type II ANOVA (analysis of variance) tests, and, when significant effects of developmental diet were detected, we explored the effect of diet further using post hoc Tukey tests. Because developmental diet might influence behaviour through effects on body mass, we first analysed the influence of developmental diet on wet and dry body mass using linear models. For aggressive behaviour that showed a significant response to focal or rival developmental diet, we further explored whether body mass had an effect on aggression above and beyond that of developmental diet, using simplified models including only focal or rival diet (depending on which had shown an effect in original models), and the corresponding mass, conducting sequential sum of squares analysis (type I ANOVA) to test the effect of mass after the main effects of developmental diet had been accounted for. We followed this by testing for effects of mass on these behaviours within each diet treatment group.

\section{Results}

\section{Developmental diet influenced adult mass}

As expected, we found that developmental diet influenced adult mass (wet mass: $\mathrm{F}_{2,373}=56.4, \mathrm{p}<0.0001$; dry mass: $\mathrm{F}_{2,370}=60.2, \mathrm{P}<0.0001$; Fig. 1). Post hoc tests revealed that low-resource males were significantly lighter than medium and high-resource males (L-M comparisons: wet mass $\mathrm{t}=-8.3, \mathrm{p}<0.0001$, dry mass $=\mathrm{t}=-8.2, \mathrm{p}<0.0001$; $\mathrm{L}-\mathrm{H}$ comparison: wet mass $\mathrm{t}=-9.9, \mathrm{p}<0.0001$, dry mass $=\mathrm{t}=-10.4, \mathrm{p}<0.0001)$, which did not differ in mass (wet mass $\mathrm{t}=1.7, \mathrm{p}=0.211$; dry mass $\mathrm{t}=-2.2, \mathrm{p}=0.067$ ), with a $12 \%$ reduction in both wet and dry mass between high- and low-resource males. These results demonstrate that males responded to our diet treatments.

\section{A resource-poor developmental diet reduced some aspects of aggression}

A focal male's developmental diet influenced his likelihood of lunging (Table 1; Fig. 2a). Post hoc analyses revealed that high-resource males were more likely to lunge than lowand medium-resource males (M-H comparison: $\mathrm{z}=-2.4$, $\mathrm{p}=0.048 ; \mathrm{L}-\mathrm{H}$ comparison: $\mathrm{z}=-2.5, \mathrm{p}=0.030)$, but low and medium-resource males did not differ $(\mathrm{z}=-0.2$, 
Fig. 1 The wet mass (A) and dry mass $(\mathbf{B})$ of adult males depending on their developmental diet. Black points show means; 'violin' areas represent the shape of the distribution; black bars show $95 \%$ confidence intervals

Table 1 The influence of focal and rival developmental diet and their interaction on focal aggressive behaviours
Fig. 2 The influence of focal and rival developmental diet on focal male lunging probability (A), number of lunges (within males that displayed lunging, B), total aggression duration (in seconds, back-transformed from square root transformation, $\mathbf{C}$ ) and wing threat number (D). Trials were $15 \mathrm{~min}$ long. Grey bars represent $95 \%$ confidence intervals
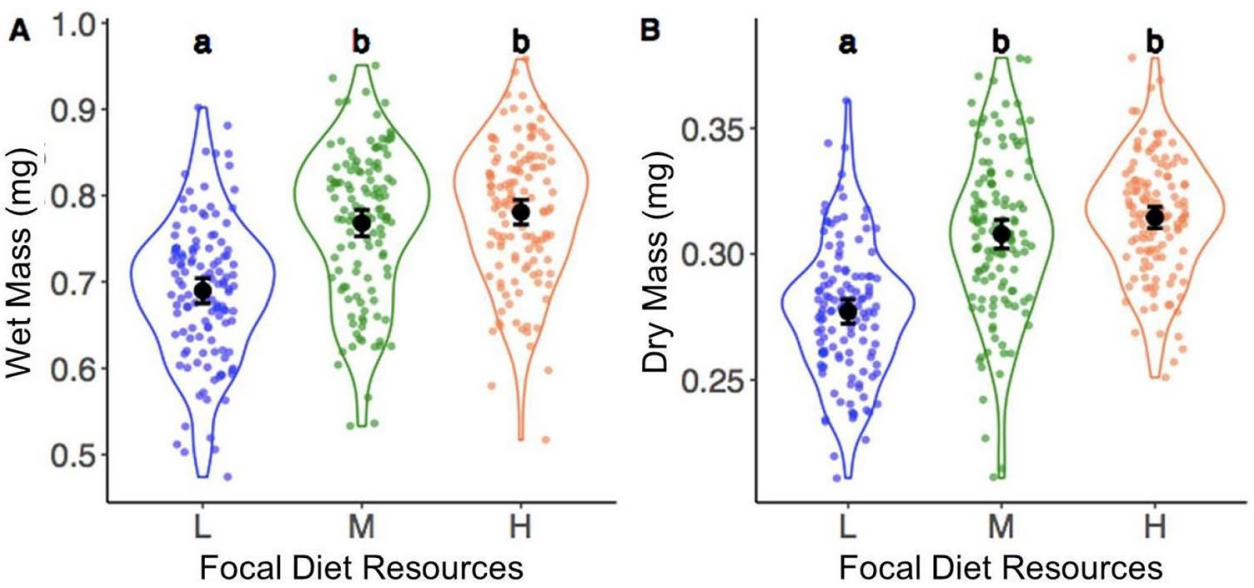

\begin{tabular}{llll}
\hline Behaviour & Focal diet & Rival diet & Focal diet $\mathrm{x}$ rival diet \\
\hline Lunging probability & $\chi_{2,174}^{2}=8.1 \mathbf{p}=\mathbf{0 . 0 1 8}$ & $\chi_{2,174}^{2}=4.1 \mathrm{p}=0.130$ & $\chi_{4,174}^{2}=1.1 \mathrm{p}=0.90$ \\
$\begin{array}{c}\text { Lunge number (amongst } \\
\quad \text { flies that lunged) }\end{array}$ & $\chi_{2,88}^{2}=4.1 \mathrm{p}=0.132$ & $\chi_{2,88}^{2}=2.1 \mathrm{p}=0.335$ & $\chi_{4,88}^{2}=6.5 \mathrm{p}=0.162$ \\
Wing threat duration & $\chi_{2,174}^{2}=8.0 \mathbf{p}=\mathbf{0 . 0 1 8}$ & $\chi_{2,174}^{2}=0.6 \mathrm{p}=0.759$ & $\chi_{4,174}^{2}=10.6 \mathbf{p}=\mathbf{0 . 0 3 2}$ \\
Total aggression duration & $\mathrm{F}_{2,174}=1.0 \mathrm{p}=0.364$ & $\mathrm{~F}_{2,174}=0.03 \mathrm{p}=0.968$ & $\mathrm{~F}_{4,174}=1.3 \mathrm{p}=0.273$ \\
\hline
\end{tabular}

Bold values indicate significance at $\alpha=0.05$
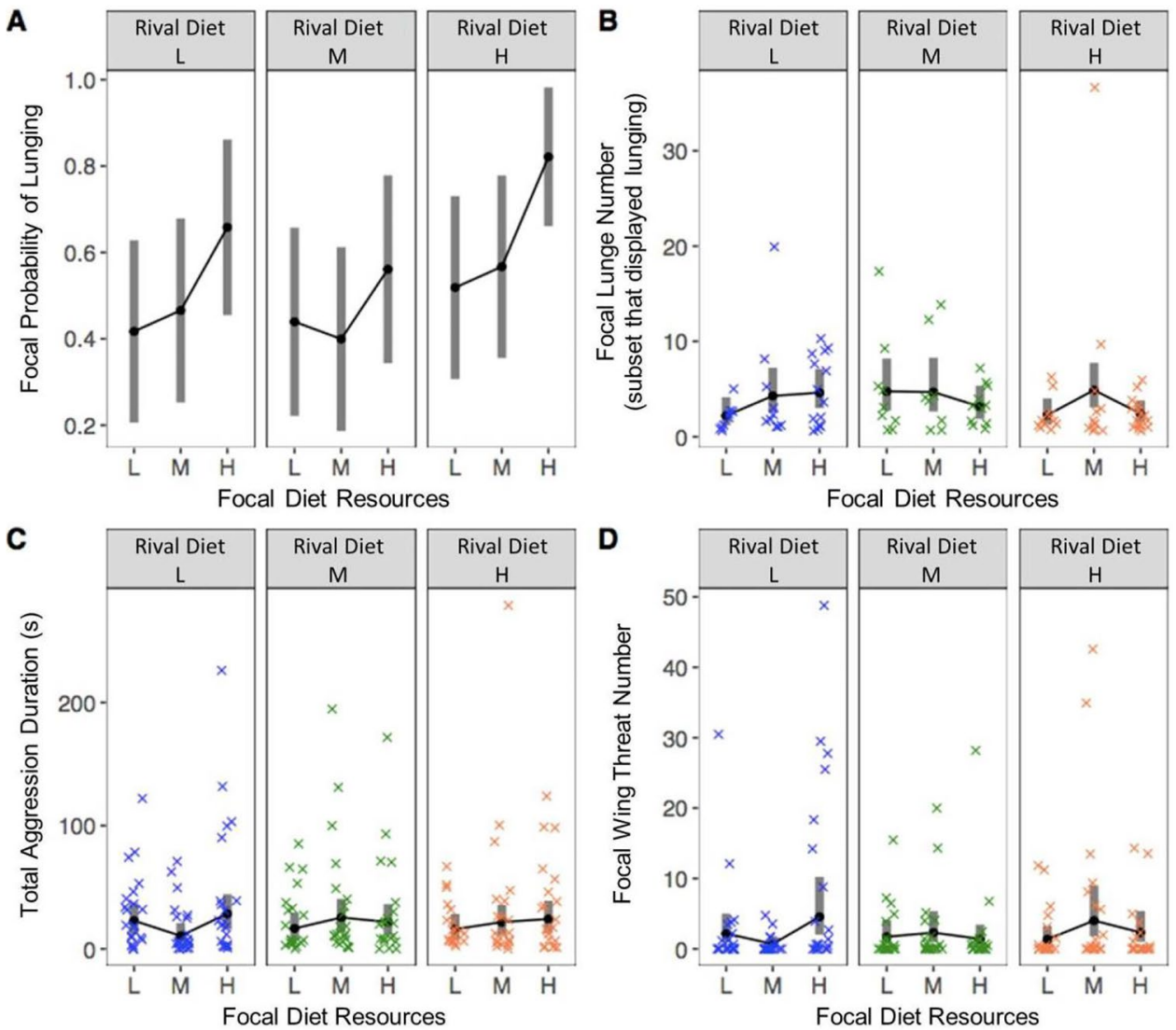
$\mathrm{p}=0.976$ ). However, in the subset of males that displayed lunging, focal male diet did not influence the number of lunges displayed (Table 1; Fig. 2b). We found no effect of a rival male's developmental diet on focal male lunging, nor evidence of an interaction between a male's developmental diet and that of his rival (Table 1; Fig. 2).

Our data do not support the hypothesis that high-resource males lunge more often solely as a consequence of their larger body mass, as focal male lunging probability was not related to focal male mass $\left(\chi^{2}{ }_{1}=0.02, \mathrm{p}=0.886\right)$, and sequential sums of squares analysis revealed no further effect of focal body mass after focal male developmental diet was accounted for (wet mass $\mathrm{F}_{1,179}=0.2, \mathrm{p}=0.653$; dry mass $\mathrm{F}_{1,179}=1.91$, $\mathrm{p}=0.168)$. Within the low-resource treatment, focal males of a lower dry mass were less likely to lunge $\left(\chi_{1,54}^{2}=6.4\right.$, $p=0.011)$, although there was no within-treatment effect of wet mass $\left(\chi_{1,54}^{2}=2.2, p=0.138\right)$. Within medium and high-resource treatments, focal mass had no influence on lunge probability (medium-resource: dry mass $\chi_{1,55}^{2}=1.4$, $\mathrm{p}=0.243$; wet mass $\chi_{1,55}^{2}=2.4, \mathrm{p}=0.118$; high-resource dry mass $\chi_{1,54}^{2}=0.6, p=0.443$; wet mass $\chi_{1,54}^{2}=0.1, p=0.814$ ).

We found no detectable effect of the developmental diet of focal and rival males on total aggressive behaviour or on chasing and fencing (Table 1; supplementary Fig. 1). Because high-resource males lunged more often but did not display more total aggression, we wondered whether highnutrition males performed other aggressive behaviours less frequently. However, we found no significant effect of developmental diet on other aggressive behaviours (Table 1; supplementary table 5; supplementary Fig. 1). Some behaviours varied with time and day (supplementary table 6), but treatments were dispersed across times and days and models included time and day to control for this variation.

Males of a high-resource developmental diet showed increased wing threat compared with males that developed on a lower-resource diet, but only when paired with rivals of a low-resource diet (Table 1; Fig. 2c). This pattern was not explained by differences in mass, as we found no effects of focal or rival male mass on wing threat number (focal male mass: $\chi_{1,176}^{2}=0.0, p=0.892$; rival male mass: $\chi_{1,176}^{2}=0.0$, $\mathrm{p}=0.862)$ and no interaction between focal and rival mass $\left(\chi_{1,176}^{2}=0.1 \mathrm{p}=0.803\right)$. Furthermore, sequential sums of squares analysis revealed no interaction between focal and rival body mass after focal and rival developmental diet were accounted for (interaction between focal and rival wet mass: $\mathrm{F}_{1,170}=0.2, \mathrm{p}=0.648$; interaction between focal and rival dry mass: $\mathrm{F}_{1,168}=0.5, \mathrm{p}=0.472$ ).

Threat displays can represent strategies to settle contests without costly escalation. However, we found no evidence that wing threat reduced escalated fighting, as there was a positive correlation between lunge number and wing threat number (Kendall's rank correlation $\tau=0.3, z=5.5$, p $<0.0001$; supplementary Fig. 2).
The influence of developmental diet on lunging and wing threat could not be explained by differences in locomotion, as we detected no differences in locomotion duration related to developmental diet (focal diet $\mathrm{F}_{2,174}=1.4, \mathrm{p}=0.240$; rival $\operatorname{diet} \mathrm{F}_{2,174}=2.6, \mathrm{p}=0.079$; supplementary Fig. 3).

\section{The developmental diet of rivals influenced aggression performed on the food patch}

We investigated how aggression related to access to the food patch because food patches represent valuable breeding sites for male D. melanogaster. We detected no effect of developmental diet on the time focal males spent on the food patch (focal diet: $\mathrm{F}_{2,174}=0.6, \mathrm{p}=0.552$, rival diet: $\mathrm{F}_{2,174}=0.1$, $\mathrm{p}=0.867$, interaction: $\mathrm{F}_{4,174}=0.8, \mathrm{p}=0.519$ ). However, focal males performed relatively more of their aggression on the food patch (as opposed to off the food patch) when competing against rivals of a low-resource developmental diet $\left(\chi_{2,168}^{2}=18.9, p<0.0001 ;\right.$ Fig. 3$)$, but neither focal diet nor the interaction between focal and rival diet had a detectable effect (focal nutrition: $\chi_{2,168}^{2}=3.4, p=0.182$; interaction: $\left.\chi_{4,168}^{2}=8.1, \mathrm{p}=0.089\right)$. Although focal males displayed relatively more of their aggression on the food patch as rival mass decreased $\left(\chi_{1,172}^{2}=5.3, \mathrm{P}=0.021\right.$; supplementary Fig. 4), sequential sums of squares analysis revealed no further effect of rival mass after rival developmental diet was accounted for (rival wet mass $\mathrm{F}_{1,178}=0.8, \mathrm{p}=0.474$; rival dry mass $\mathrm{F}_{1,176}=0.2, \mathrm{p}=0.849$ ).

\section{Aggression levels are not correlated within pairs}

Because rival behaviour might influence focal male behaviour, we examined the relationship between the two. Chisquare analysis revealed that the probabilities of a focal and rival male performing at least one lunge are not independent $\left(\chi_{1}^{2}=18.5, p<0.001\right)$. We found some evidence that diet influences this relationship, because although focal and rival male lunging was related in the low- and mediumresource focal developmental diet treatments (low: $\chi_{1}{ }_{1}=7.7$, $\mathrm{p}=0.006$; medium: $\left.\chi^{2}{ }_{1}=4.5, \mathrm{p}=0.034\right)$, we found no relationship the high-resource focal developmental diet treatment $\left(\chi_{1}^{2}=2.3, p=0.126\right)$. Amongst those focal males that lunged, rival lunge number did not influence focal lunge number $\left(\chi_{1,95}^{2}=0.5, p=0.470\right)$. Furthermore, the total duration of focal male aggression was not related to the total duration of rival aggression $\left(\mathrm{F}_{1,181}=1.2, \mathrm{p}=0.277\right)$, and this was not influenced by differences in developmental diet (duration of rival aggression $\times$ focal diet interaction: $\mathrm{F}_{2,165}=0.2, \mathrm{p}=0.855$, duration of rival aggression $\times$ rival diet interaction: $\mathrm{F}_{2,165}=0.3, \mathrm{p}=0.724$ ). 
Fig. 3 The influence of focal and rival developmental diet on the proportion of aggression the focal male performed on the food, relative to off the food. Grey bars represent $95 \%$ confidence intervals

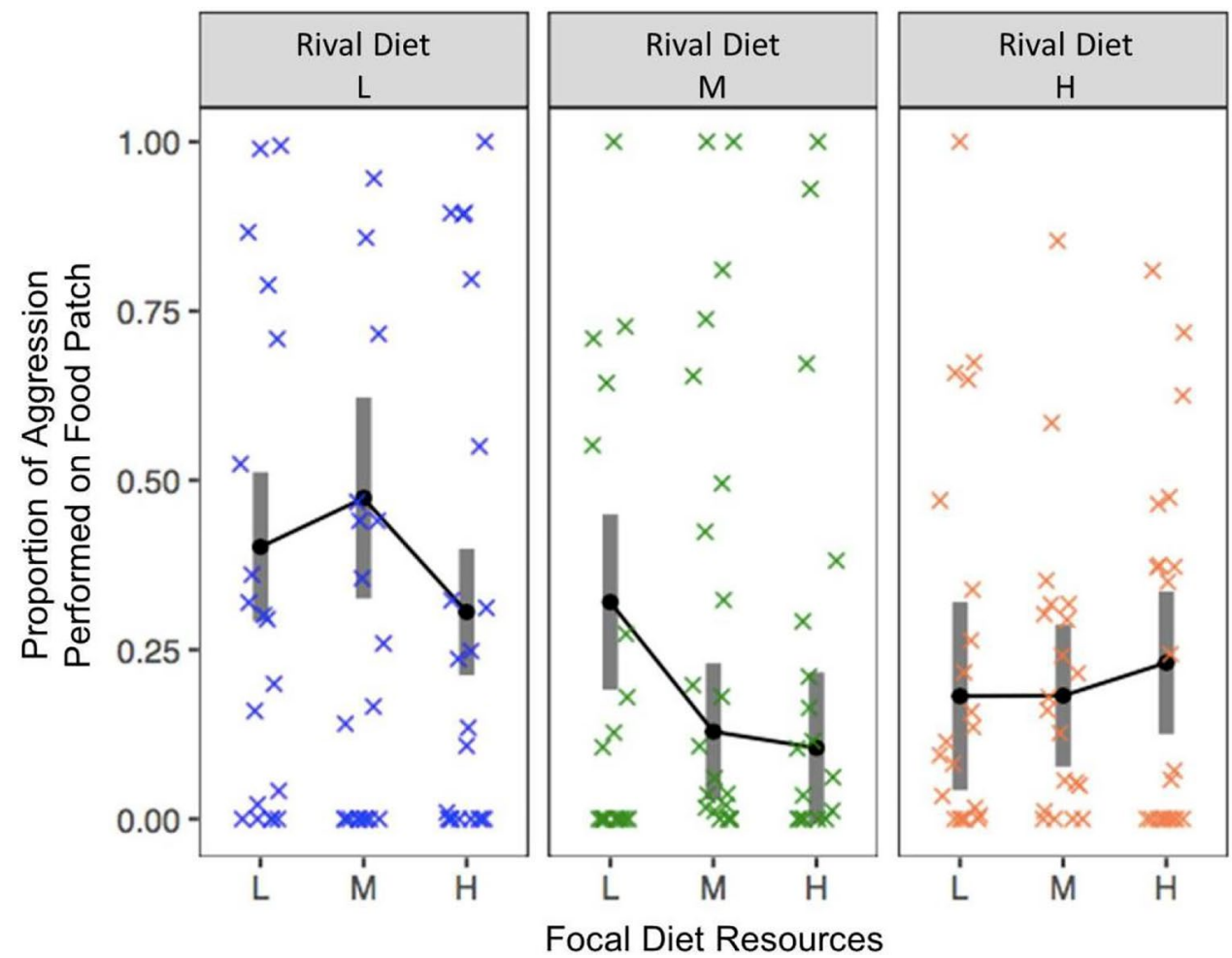

\section{Discussion}

We found that developmental diet influenced adult male aggression in D. melanogaster: a low-resource developmental diet reduced the likelihood of aggressive lunging (against all rivals) and wing threats (against rivals of low-resource diet). The influence of developmental diet on aggression appears above and beyond any influence of body mass. The developmental diet of rival males influenced where males performed aggression: focal males concentrated their aggression to the food patch when competing with low-resource rivals, suggesting that males may be better able to access food resources against nutritionally poorer rivals. Our findings suggest that adult $D$. melanogaster alter aggressive behaviour in light of their own developmental diet, through factors distinct from body mass, and that the nutritional experience of social partners also impacts contest characteristics.

The hypothesis that a resource-poor developmental diet would restrict growth and allocation to traits that mediate aggression (Monaghan 2008) predicts that a resourcepoor developmental diet should decrease adult aggression. The reduced lunging probability of males subjected to low resource during development supports this prediction. However, although a low-resource developmental diet reduced adult body mass, in line with previous findings (Zikovitz and Agrawal 2013), differences in body mass did not explain the relationship with aggression, over and above the effect of developmental diet. This suggests that the relationship is mediated through mass-independent effects of diet, such as changes to internal state and energy budget. There was substantial mass variation amongst treatments, suggesting that insufficient variation did not explain the absence of mass effects beyond diet effects. Our results suggest that underlying variation in the developmental environment might explain the positive association between mass and aggression found in both male and female $D$. melanogaster (Hoffman 1987; Markow 1988; Hoyer et al. 2008; Bath et al. 2018) as well as other invertebrates (Shackleton et al. 2005; Brown et al. 2007) and vertebrates (reviewed by Briffa and Sneddon 2007).

Developmental diet might play a larger role in determining aggression than body mass per se does because diet can influence a range of physiological factors including resource allocation, energy levels and the relative growth of different traits. Indeed, in male Drosophila, developmental diet can have wide-ranging impacts, with resource-poor developmental diets reducing a male's ability to transfer sperm and induce a refractory state in mates (McGraw et al. 2007), reducing his courtship success (Sharp and Agrawal 2009; Morimoto et al. 2016; Wigby et al. 2016), and reducing his success in post-copulatory sperm competition (Bangham et al. 2002; Morimoto et al. 2016). These effects can also be independent of the influence of diet on mass (McGraw et al. 2007). Furthermore, in other species, nutrition-induced correlates of condition, such as resting metabolic rate and 
energy reserves, can better predict aggression than body mass does (e.g. in the freshwater prawn Macrobrachium rosenbergii, Brown et al. 2003 swordtail Xiphophorus helleri, Royle et al. 2005, and damselfly Calopterya splendens xanthostama, Plaistow and Siva-Jothy 1996). If developmental nutrition does cause differences in physiology, then males that develop on resource-poor diets might adopt alternative strategies to maximise fitness returns from their limited energy reserves, rather than engaging in contests they are likely to lose.

We found that some aspects of male aggression-including chasing and fencing_-did not show the same response to developmental diet as did lunging. The high intensity of lunging might make it more sensitive to developmental resource levels than less intense aggressive behaviours. Furthermore, the results suggest that developmental diet influences the propensity to engage in high-intensity lunging, but has less influence on the number of lunges required to resolve a contest. These results highlight how measuring a single aggressive behaviour might not capture the full picture of how ecological factors influence aggression (Chen et al. 2002; Alekseyenko et al. 2010; Certel and Kravitz 2012). Our finding that developmental diet has varying influences on different aspects of aggression is consistent with results for mating behaviour in D. melanogaster. The developmental environment influences several male sexual traits (as described above; Bangham et al. 2002; McGraw et al. 2007; Wigby et al. 2016), but other sexual traits such as sperm length (Amitin and Pitnick 2007) and mating rate, duration and latency (Lefranc and Bundgaard 2000; Edward and Chapman 2012) show little or no sensitivity. Thus, different aspects of multifaceted behaviours, such as aggressive and sexual behaviours, might be free to vary independently, allowing fine-tuned responses to ecological cues. Interestingly, nutritionally stressful developmental conditions can increase larval cannibalism-a potential form of developmental aggression-in D. melanogaster (Vijendravarma et al. 2013). When taken with our finding of reduced adult aggressive lunging after a resource-poor developmental diet, this suggests contrasting short-term and long-term effects of developmental nutrition on aggressive behaviours.

Nutrient quality and quantity can signal the nature of the prevailing social environment, providing information about mates, rivals and the costs and benefits of adult behavioural strategies (Enquist and Leimar 1987; Elias et al. 2010). Such information can influence behavioural motivation and resource valuation and prime individuals to cope with similar conditions as adults (Wigby et al. 2016). Our findings do not support the prediction that low-resource developmental diets increase aggression through increased resource valuation and motivation to compete aggressively for access to food patches (Elias et al. 2010; Bath et al. 2018). Alternatively, because moderate nutritional deprivation (i.e. that does not reach the severity of starvation) can increase lifespan in D. melanogaster (Pletcher et al. 2002; Klepsatel et al. 2018) and other species (Mair and Dillin 2008), flies that develop under resource-poor conditions might delay reproductive effort and have reduced motivation for early-life contests over breeding territories (Dow and von Schilcher 1975; Hoffman 1987; Hoffmann 1987; Chen et al. 2002). Future studies that explore the relationship between developmental diet, lifespan and aggression would be illuminating. The benefit of behavioural plasticity in response to environmental variation decreases as the duration between cue detection and the performance of the behavioural strategy increases (Fusco and Minelli 2010; Bretman et al. 2011a, $2011 \mathrm{~b}$ ). Hence, changes in resource valuation in response to adult nutrition might override any developmental nutritional experience (Edmunds et al. 2021). Further studies that consider the influence of adult nutrition on aggression would help resolve this question.

Surprisingly, males did not vary their level of direct physical fighting in response to the developmental diet of their rival. Contest theory suggests that physical fighting should be used sparingly against rivals of superior condition (Maynard Smith and Parker 1976; Bishop and Cannings 1978; Hammerstein and Parker 1982; Enquist and Leimar 1983; Leimar and Enquist 1984; Briffa and Sneddon 2007). Previous studies report that both male and female D. melanogaster regulate their aggression in response to opponent body mass (Hoffman 1987; Bath et al. 2018). However, the diet-induced variation in body mass in this study was relatively small and more representative of natural variation (e.g. a $12 \%$ difference in mass between low- and highdevelopmental diet males in these experiments, versus a 50\% difference in Hoffmann, 1987). Because losing fights in $D$. melanogaster seldom results in direct physical damage to structures such as the wings (Guo and Dukas 2020), fighting behaviour might be less sensitive to small differences in rival conditions in this species. Our results support the view that fighting is instead primarily determined by an individual's own developmental diet.

However, focal individuals did respond to the developmental diet of their rival in the relative amount of aggression performed on the food patch and in threat behaviour. Food patches can represent both nutritional sources and breeding sites (Markow 1988; Lim et al. 2014), so this result suggests that developmental diet and subsequent adult mass of a rival might influence a male's ability to dominate access to these sites. Threat displays can allow individuals to assess or intimidate rivals without engaging in costly fights (Clutton-Brock and Albon 1979; Logue et al. 2010). Interestingly, recent work in D. melanogaster has demonstrated that populations evolved on a low-carbohydrate, high-protein diet in one laboratory favour wing threats over lunges in establishing dominance, whereas those evolved on 
a higher-carbohydrate, low-protein diet in another laboratory display more lunges, suggesting these two behaviours might represent alternate strategies (Legros et al. 2021). However, it is not obvious from our results that wing threats functioned as a strategy to avoid lunging. At face value, our findings that males that developed on a high-resource diet performed both more wing threats and more aggressive lunging do not suggest that the use of wing threats reduced lunging, but it is not possible to tell from our data if flies would have lunged more often had they not used these threat displays. Furthermore, it is possible that in natural settings, or in the large population cages in which these males recently evolved, threat displays by high-resource males might cause low-resource rivals to flee, avoiding escalated conflict, but this was not possible in our observation chambers.

Previous research has demonstrated that multiple aspects of the D. melanogaster diet can influence male fighting and reproductive behaviour throughout life. For example, excess saturated and trans-fatty acids in the adult diet can induce a more aggressive state (Meichtry et al. 2020), food deprivation increases male aggression (Edmunds et al. 2021), and developmental nutrition can influence post-copulatory male-male competition (McGraw et al. 2007). Our results add to this growing body of literature by providing evidence of an influence of developmental yeast levels on aggression. We demonstrate that, under our experimental conditions, an individual's direct aggression is primarily determined by its own developmental diet, but the developmental experience of the rival influences threat behaviour and the defence of food patches during contests. Our findings contribute to the view that early-life experiences, particularly nutritional experience, shape behaviour throughout life (Monaghan 2008; Gluckman et al. 2016). Given the extensive molecular genetic approaches available in this model organism (Asahina 2017), our results offer the opportunity for future exploration of the mechanisms underlying nutritional regulation of aggression that could be applied on a broader taxonomic scale. In humans, the balance of nutrients received during childhood can influence aggressive behaviours later in life (Liu et al. 2004; Galler et al. 2012; Collie et al. 2020). Uncovering the ecological factors that determine aggression can help us understand variation in antagonistic behavioural strategies and predict social dynamics, and, in a human context, might help to limit the negative consequences of aggression.

Supplementary Information The online version contains supplementary material available at https://doi.org/10.1007/s00265-021-03050-z.

Acknowledgements DE was funded by the Biotechnology and Biological Sciences Research Council Doctoral Training Partnership (BBSRC DTP). SW was supported by a David Phillips Fellowship from the BBSRC (BB/K014544/1). JCP was funded by a fellowship from the Natural Environment Research Council (NERC; NE/P017193/1) and a
Jesus College Major Research Grant. The authors thank Rachel LoosBennett for laboratory assistance and three anonymous reviewers for suggestions that improved the manuscript.

Author contribution DE, SW and JCP conceived the ideas and designed the methodology; DE collected the data; DE, SW and JCP analysed the data; DE drafted the initial version of the manuscript and all authors contributed to later versions of the manuscript.

Funding DE was funded by the Biotechnology and Biological Sciences Research Council Doctoral Training Partnership (BBSRC DTP). SW was supported by a David Phillips Fellowship from the BBSRC (BB/ K014544/1). JCP was funded by a fellowship from the Natural Environment Research Council (NERC; NE/P017193/1) and a Jesus College Major Research Grant.

Data availability Data are available as supplementary material.

\section{Declarations}

Conflict of interest The authors declare no competing interests.

Open Access This article is licensed under a Creative Commons Attribution 4.0 International License, which permits use, sharing, adaptation, distribution and reproduction in any medium or format, as long as you give appropriate credit to the original author(s) and the source, provide a link to the Creative Commons licence, and indicate if changes were made. The images or other third party material in this article are included in the article's Creative Commons licence, unless indicated otherwise in a credit line to the material. If material is not included in the article's Creative Commons licence and your intended use is not permitted by statutory regulation or exceeds the permitted use, you will need to obtain permission directly from the copyright holder. To view a copy of this licence, visit http://creativecommons.org/licenses/by/4.0/.

\section{References}

Alekseyenko OV, Lee C, Kravitz EA (2010) Targeted manipulation of serotonergic neurotransmission affects the escalation of aggression in adult male Drosophila melanogaster. PLoS ONE 5(5):e10806

Amitin EG, Pitnick S (2007) Influence of developmental environment on male- and female-mediated sperm precedence in Drosophila melanogaster. J Evol Biol 20:381-391

Arnott G, Elwood RW (2008) Information gathering and decision making about resource value in animal contests. Anim Behav 76:529-542

Asahina K (2017) Neuromodulation and strategic action choice in Drosophila aggression. Annual Review of Neuroscience 40:51-75

Asahina K, Watanabe K, Duistermars BJ, Hoopfer E, Gonzalez CR, Eyjolfsdottir EA, Perona P, Anderson DJ (2014) Tachykininexpressing neurons control male-specific aggressive arousal in Drosophila. Cell 156:221-235

Bangham J, Chapman T, Partridge L (2002) Effects of body size, accessory gland and testis size on pre- and postcopulatory success in Drosophila melanogaster. Anim Behav 64:915-921

Bath E, Edmunds D, Norman J, Atkins C, Harper L, Rostant WG, Chapman T, Wigby S, Perry JC (2021) Sex ratio and the evolution of aggression in fruit flies. Proc Roy Soc B 288:20203053

Bath E, Morimoto J, Wigby S (2018) The developmental environment modulates mating-induced aggression and fighting success in adult female Drosophila. Func Ecol 32:2542-2552 
Belenioti M, Chaniotakis N (2020) Aggressive behaviour of Drosophila suzukii in relation to environmental and social factors. Scientific Reports 10:7898

Bishop DT, Cannings C (1978) A generalized war of attrition. J Theor Biol 70:85-124

Blanchard DC, Blanchard RJ (2003) What can animal aggression research tell us about human aggression? Hormones and Behavior 44:171-177

Blumstein DT, Daniel JC (2007) Quantifying behavior the JWatcher way. Sinauer

Boggs CL (1981) Nutritional and life-history determinants of resource allocation in holometabolous insects. Am Nat 117:692-709

Bretman A, Gage MJ, Chapman T (2011a) Quick-change artists: male plastic behavioural responses to rivals. Trends Ecol Evol 26:467-473

Bretman A, Westmancoat JD, Gage MJ, Chapman T (2011b) Males use multiple, redundant cues to detect mating rivals. Curr Biol 21:617-622

Briffa M, Sneddon LU (2007) Physiological constraints on contest behaviour. Func Ecol 21:627-637

Bross TG, Rogina B, Helfand SL (2005) Behavioral, physical, and demographic changes in Drosophila populations through dietary restriction. Aging Cell 4:309-317

Brown JH, Ross B, McCauley S, Dance S, Taylor AC, Huntingford FA (2003) Resting metabolic rate and social status in juvenile giant freshwater prawns, Macrobrachium rosenbergii. Marine and Freshwater Behaviour and Physiology 36:31-40

Brown WD, Chimenti AJ, Siebert JR (2007) The payoff of fighting in house crickets: motivational asymmetry increases male aggression and mating success. Ethology 113:457-465

Carazo P, Perry JC, Johnson F, Pizzari T, Wigby S (2015) Related male Drosophila melanogaster reared together as larvae fight less and sire longer lived daughters. Ecol Evol 5:2787-2797

Carvalho GB, Kapahi P, Anderson DJ, Benzer S (2006) Allocrine modulation of feeding behavior by the sex peptide of Drosophila. Curr Biol 16:692-696

Certel SJ, Kravitz EA (2012) Scoring and analyzing aggression in Drosophila. Cold Spring Harbor Protocols 2012:319-325

Chen S, Lee AY, Bowens NM, Huber R, Kravitz EA (2002) Fighting fruit flies: a model system for the study of aggression. Proc Natl Acad Sci USA 99:5664-5668

Clutton-Brock TH (1984) Reproductive effort and terminal investment in iteroparous animals. Am Nat 123:212-229

Clutton-Brock TH, Albon SD (1979) The roaring of red deer and the evolution of honest advertisement. Behaviour 69:145-170

Colasurdo N, Gelinas Y, Despland E (2009) Larval nutrition affects life history traits in a capital breeding moth. J Exp Biol 212:1794-1800

Collie J, Granela O, Brown EB, Keene AC (2020) Aggression is induced by resource limitation in the monarch caterpillar. iScience 23:101791

Dierick HA (2007) A method for quantifying aggression in male Drosophila melanogaster. Nature Protocols 2:2712-2718

Dochtermann NA, Schwab T, Sih A (2015) The contribution of additive genetic variation to personality variation: heritability of personality. Proc Roy Soc B 282:20142201

Dow MA, von Schilcher F (1975) Aggression and mating success in Drosophila melanogaster. Nature 254:511-512

Edmunds D, Wigby S, Perry JC (2021) 'Hangry' Drosophila: food deprivation increases male aggression. Anim Behav 177:183-190

Edward DA, Chapman T (2012) Sex-specific effects of developmental environment on reproductive trait expression in Drosophila melanogaster. Ecol Evol 2:1362-1370
Elias DO, Botero CA, Andrade MC, Mason AC, Kasumovic MM (2010) High resource valuation fuels "desperado" fighting tactics in female jumping spiders. Behav Ecol 21:868-875

Enquist M, Leimar O (1983) Evolution of fighting behaviour: decision rules and assessment of relative strength. $\mathrm{J}$ Theor Biol 102:387-410

Enquist M, Leimar O (1987) Evolution of fighting behaviour: the effect of variation in resource value. J Theor Biol 127:187-205

Fusco G, Minelli A (2010) Phenotypic plasticity in development and evolution: facts and concepts. Phil Trans Roy Soc 365:547-556

Galler JR, Bryce CP, Waber DP, Hock RS, Harrison R, Eaglesfield GD, Fitzmaurice G (2012) Infant malnutrition predicts conduct problems in adolescents. Nutritional Neuroscience 15:186-192

Gebhardt MD, Stearns SC (1993) Phenotypic plasticity for life history traits in Drosophila melanogaster. I. Effect on phenotypic and environmental correlations. J Evol Biol 6:1-16

Georgiev AV, Klimczuk AC, Traficonte DM, Maestripieri D (2013) When violence pays: a cost-benefit analysis of aggressive behavior in animals and humans. Evolutionary Psychology 11:678-699

Gluckman PD, Buklijas T, Hanson MA (2016) The developmental origins of health and disease $(\mathrm{DOHaD})$ concept: past, present, and future. In: Rosenfeld CS (ed) The Epigenome and Developmental Origins of Health and Disease. Elsevier Science, London, UK, pp 1-15

Good TP, Tatar M (2001) Age-specific mortality and reproduction respond to adult dietary restriction in Drosophila melanogaster. J Insect Physiol 47:1467-1473

Grover CD, Kay AD, Monson JA, Marsch TC, Holway DA (2007) Linking nutrition and behavioural dominance: carbohydrate scarcity limits aggression and activity in Argentine ants. Proc Roy Soc B 274:2951-2957

Gruber C, Tulonen J, Kortet R, Hirvonen H (2016) Resource availability and predation risk influence contest behavior and dominance hierarchies in crayfish. Behav Ecol Soc 70:1305-1317

Guo X, Dukas R (2020) The cost of aggression in an animal without weapons. Ethology 126:24-31

Haley MP (1994) Resource-holding power asymmetries, the prior residence effect, and reproductive payoffs in male northern elephant seal fights. Behav Ecol Soc 34:427-434

Hammerstein P, Parker GA (1982) The asymmetric war of attrition. J Theor Biol 96:647-682

Han CS, Dingemanse NJ (2017) You are what you eat: diet shapes body composition, personality and behavioural stability. BMC Evol Biol 17:8

Harrison XA, Donaldson L, Correa-Cano ME, Evans J, Fisher DN, Goodwin CED, Robinson BS, Hodgson DJ, Inger R (2018) A brief introduction to mixed effects modelling and multi-model inference in ecology. PeerJ 6:e4794

Hoffman AA (1987) Territorial encounters between Drosophila males of different sizes. Anim Behav 35:1899-1901

Hoffman AA, Cacoyianni Z (1990) Territoriality in Drosophila melanogaster as a conditional strategy. Anim Behav 40:526-537

Hoffmann AA (1987) A laboratory study of male territoriality in the sibling species Drosophila melanogaster and D. simulans. Anim Behav 35:807-818

Hoyer SC, Eckart A, Herrel A, Zars T, Fischer SA, Hardie SL, Heisenberg M (2008) Octopamine in male aggression of Drosophila. Curr Biol 18:159-167

Huntingford F, Tamilselvan P, Jenjan H (2012) Why do some fish fight more than others? Physiological and Biochemical Zoology 85:585-593

Jang T, Lee KP (2018) Comparing the impacts of macronutrients on life-history traits in larval and adult Drosophila melanogaster: the use of nutritional geometry and chemically defined diets. J Exp Biol 221:jeb181115

Johnsen TS, Zuk M (1995) Testosterone and aggression in male red jungle fowl. Hormones and Behavior 29:593-598 
Klepsatel P, Prochazka E, Galikova M (2018) Crowding of Drosophila larvae affects lifespan and other life-history traits via reduced availability of dietary yeast. Experimental Gerontology 110:298-308

Krams IA, Krama T, Moore FR, Rantala MJ, Mand R, Mierauskas P, Mand M (2015) Resource availability as a proxy for terminal investment in a beetle. Oecologia 178:339-345

Kravitz EA, Fernandez MP (2015) Aggression in Drosophila. Behavioral Neuroscience 129:549-563

Lefranc A, Bundgaard J (2000) The influence of male and female body size on copulation duration and fecundity in Drosophila melanogaster. Hereditas 132:243-247

Legros J, Tang G, Gautrais J, Fernandez MP, Trannoy S (2021) Longterm dietary restriction leads to development of alternative fighting strategies. Frontiers in behavioral neuroscience 14:599676

Leimar O, Enquist M (1984) Effects of asymmetries in owner-intruder conflicts. J Theor Biol 111:475-491

Lihoreau M, Buhl J, Charleston MA, Sword GA, Raubenheimer D, Simpson SJ (2015) Nutritional ecology beyond the individual: a conceptual framework for integrating nutrition and social interactions. Ecol Lett 18:273-286

Lim RS, Eyjolfsdottir E, Shin E, Perona P, Anderson DJ (2014) How food controls aggression in Drosophila. PLoS ONE 9:e105626

Liu J, Raine A, Venables PH, Mednick SA (2004) Malnutrition at age 3 years and externalizing behavior problems at ages 8,11 , and 17 years. American Journal of Psychiatry 161:2005-2013

Logue DM, Abiola IO, Rains D, Bailey NW, Zuk M, Cade WH (2010) Does signalling mitigate the cost of agonistic interactions? A test in a cricket that has lost its song. Proc Roy Soc B 277:2571-2575

Mair W, Dillin A (2008) Aging and survival: the genetics of life span extension by dietary restriction. Annual Review of Biochemistry 77:727-754

Markow TA (1988) Reproductive behavior of Drosophila melanogaster and D. nigrospiracula in the field and in the laboratory. Journal of Comparative Psychology 102:169-173

Maynard Smith J, Parker GA (1976) The logic of asymmetric contests. Anim Behav 24:159-175

McGraw LA, Fiumera AC, Ramakrishnan M, Madhavarapu S, Clark AG, Wolfner MF (2007) Larval rearing environment affects several postcopulatory traits in Drosophila melanogaster. Biol Lett 3:607-610

Meichtry LB, Poetini MR, Dahleh MMM, Araujo SM, Musachio EAS, Bortolotto VC, de Freitas CS, Somacal S, Emanuelli T, Gayer MC, Roehrs R, Guerra GP, Prigol M (2020) Addition of saturated and trans-fatty acids to the diet induces depressive and anxiety-like behaviors in Drosophila melanogaster. Neuroscience 443:164-175

Moatt JP, Nakagawa S, Lagisz M, Walling CA (2016) The effect of dietary restriction on reproduction: a meta-analytic perspective. BMC Evol Biol 16:199

Monaghan P (2008) Early growth conditions, phenotypic development and environmental change. Phil Trans Roy Soc 363:1635-1645

Morimoto J, Pizzari T, Wigby S (2016) Developmental environment effects on sexual selection in male and female Drosophila melanogaster. PLoS ONE 11:e0154468

Neat FC, Taylor AC, Huntingford FA (1998) Proximate costs of fighting in male cichlid fish: the role of injuries and energy metabolism. Anim Behav 55:875-882

Nilsen SP, Chan YB, Huber R, Kravitz EA (2004) Gender-selective patterns of aggressive behavior in Drosophila melanogaster. Proc Natl Acad Sci USA 101:12342-12347

Parker GA (1974) Assessment strategy and the evolution of fighting behaviour. J Theor Biol 47:223-243

Penn JK, Zito MF, Kravitz EA (2010) A single social defeat reduces aggression in a highly aggressive strain of Drosophila. Proc Natl Acad Sci USA 107:12682-12686

Pillay N, Rimbach R, Rymer T (2016) Pre- and postnatal dietary protein deficiency influences anxiety, memory and social behaviour in the African striped mouse Rhabdomys dilectus chakae. Physiology and Behavior 161:38-46

Plaistow S, Siva-Jothy MT (1996) Energetic constraints and male matesecuring tactics in the damselfly Calopteryx splendens xanthostoma (Charpentier). Proc Roy Soc B 263:1233-1239

Pletcher SD, Macdonald SJ, Marguerie R, Certa U, Stearns SC, Goldstein DB, Partridge L (2002) Genome-wide transcript profiles in aging and calorically restricted Drosophila melanogaster. Curr Biol 12:712-723

Rodrigues MA, Martins NE, Balance LF, Broom LN, Dias AJ, Fernandes AS, Rodrigues F, Sucena E, Mirth CK (2015) Drosophila melanogaster larvae make nutritional choices that minimize developmental time. J Insect Physiol 81:69-80

Royle NJ, Lindstrom J, Metcalfe NB (2005) A poor start in life negatively affects dominance status in adulthood independent of body size in green swordtails Xiphophorus helleri. Proc Roy Soc B 272:1917-1922

Sang JH (1978) The nutritional requirements of Drosophila. In: Ashburner M, Wright TRF (eds) The Genetics and Biology of Drosophila. Academic Press, New York, pp 159-192

Scharf I (2016) The multifaceted effects of starvation on arthropod behaviour. Anim Behav 119:37-48

Seebacher F, Ward AJ, Wilson RS (2013) Increased aggression during pregnancy comes at a higher metabolic cost. J Exp Biol 216:771-776

Shackleton MA, Jennions MD, Hunt J (2005) Fighting success and attractiveness as predictors of male mating success in the black field cricket, Teleogryllus commodus: the effectiveness of nochoice tests. Behav Ecol Soc 58:1-8

Sharp NP, Agrawal AF (2009) Sexual selection and the random union of gametes: testing for a correlation in fitness between mates in Drosophila melanogaster. Am Nat 174:613-622

Sinn DL, While GM, Wapstra E (2008) Maternal care in a social lizard: links between female aggression and offspring fitness. Anim Behav 76:1249-1257

Sluyter F, Arseneault L, Moffitt TE, Veenema AH, de Boer S, Koolhaas JM (2003) Toward an animal model for antisocial behavior: parallels between mice and humans. Behav Genet 33:563-574

Stockermans BC, Hardy IC (2013) Subjective and objective components of resource value additively increase aggression in parasitoid contests. Biol Lett 9:20130391

Tigreros N (2013) Linking nutrition and sexual selection across life stages in a model butterfly system. Func Ecol 27:145-154

Trannoy S, Penn J, Lucey K, Popovic D, Kravitz EA (2016) Short and long-lasting behavioral consequences of agonistic encounters between male Drosophila melanogaster. Proc Natl Acad Sci USA 113:4818-4823

Vijendravarma RK, Narasimha S, Kawecki TJ (2013) Predatory cannibalism in Drosophila melanogaster larvae. Nat Commun 4:1-8

Wallner B, Machatschke IH (2009) Influence of nutrition on aggression. CAB Reviews: Perspectives in Agriculture, Veterinary Science, Nutrition and Natural Resources 4:1-10

Wigby S, Perry JC, Kim Y-H, Sirot L (2016) Developmental environment mediates male seminal protein investment in Drosophila melanogaster. Func Ecol 30:410-419

Zikovitz AE, Agrawal AF (2013) The condition dependency of fitness in males and females: the fitness consequences of juvenile diet assessed in environments differing in key adult resources. Evolution 67:2849-2860

Publisher's note Springer Nature remains neutral with regard to jurisdictional claims in published maps and institutional affiliations. 